Original Article

\title{
SYNTHESIS AND UTILITY OF NEW POLYCYCLIC COMPOUNDS AS POTENTIAL ANTIMICROBIALS BASED ON CHROMENE MOIETY
}

\author{
MAHMOUD N. ABDELAZIZ1 ${ }^{1}$, EMAN S. ZARIE ${ }^{2,3 *}$, ALAADIN E. SARHAN³
}

${ }_{1}^{1}$ Faculty of Science (Girls), Chemistry Department, Al-Azhar University, Nasr City, Cairo, Egypt, ${ }^{2}$ Nanochemistry and Nanoengineering, School of Chemical Engineering, Department of Chemistry and Materials Science, Aalto University, Kemistintie 1, 00076 Aalto, Finland, ${ }^{3}$ Department of Therapeutical Chemistry, Pharmaceutical Division, National Research Centre, Dokki 12311, Giza, Egypt Email: eman.zarie@aalto.fi

Received: 28 May 2019 Revised and Accepted: 30 Jul 2019

\section{ABSTRACT}

Objective: The present research aims to synthesize some new polycyclic compounds including chromene moiety and study their antimicrobial activity.

Methods: Several new polycyclic systems including chromene scaffold incorporated with pyridine, pyrimidine, imidazopyrimidine, and imidazodiazocine were achieved via condensation reaction of chromene derivative under the proper condition with various reagents namely; cyanothioacetamide, phenyl isothiocyanate, malononitrile, carbon disulfide, benzaldehyde, triethylorthoformate, and 1,4-dichlorobutane. Moreover, a chlorodiazenyl chromene derivative was reacted with some substances possessing active- $\mathrm{CH}_{2}$-bridge such as ethyl cyanoacetate and malononitrile to end up with hydrazono compounds. Such compounds were eventually cyclized with hydrazine hydrate to form pyrazole and oxopyrazole derivatives. Moreover, compound 1 was treated with benzoyl acetone, and then followed by cyclization with malononitrile to provide the corresponding 2-amino14-(4-methoxyphenyl)-4-methy-5-phenyl-14H-benzo[5,6] chromeno [2,3H][1,6]naphthyridine-3-carbonitrile (20).

Results: The results of the antimicrobial screening in vitro revealed that the inhibition zone $(\mathrm{mm})$ of the synthesized compounds 1-3, 5 and 8 implied their optimum antibacterial activity, while the compounds 4, 6 and 9-13, 15 showed a moderate to weak antibacterial activity against multiple species of B. subtilis, $S$. aureus, E. coli and P. aeruginosa. In contrast, the compounds 1, 6, 11, 15 showed high antifungal activities against different species of A. flavinand C. albicans, while the other compounds exhibit a moderate to poor antifungal activity.

Conclusion: It is remarkable that a series of chromene derivatives synthesized by a simple and available method leads to a molecule of promising antimicrobial activity. Further research is recommended to approve the importance of polycyclic systems for various applications.

Keywords: Chromene-3-carbonitrile, Chromenes, Polycyclic compounds, Pyrimidines, Fused ring, Antimicrobial activity

(C) 2019 The Authors. Published by Innovare Academic Sciences Pvt Ltd. This is an open-access article under the CC BY license (http://creativecommons.org/licenses/by/4.0/) DOI: http://dx.doi.org/10.22159/ijpps.2019v11i9.34338

\section{INTRODUCTION}

The resistance of bacteria to antimicrobial drugs is a well-known phenomenon [1-2]. As a result, new drugs based on less toxic materials are needed and should be designed and developed. The chromene nuclei have drawn wide attention for many applications in the field of biology, chemotherapy, pharmacology, therapeutical chemistry, and materials science. Chromenes are consisting of benzene and pyrane fused ring called benzopyrans which used as starting materials for the synthesis of bioactive structures [3-25]. Since ten years ago, the chromene derivatives have been extensively used as active compounds to treat cancer [26, 27] inflammation [28], cardiovascular diseases (CVD) [29]. Moreover, they have been used as antimicrobial [30-32], antioxidant [33] antiviral [34], anthelminthic [35], anti-HIV [36], TNF- $\alpha$-inhibitor [37], estrogenic [38, 39] antitubercular [40-41] herbicidal [42], anticonvulsant, antiparkinsonian $[43,44]$, antidepressant $[45]$ and analgesic $[46,47]$ activity. Moreover, some of chromene scaffold are well known as antimicrobial drugs and their analogues such as novobiocin, chlorobiocin and coumermycin $\mathrm{Al}$ [48-50]. In the present research, we aimed to synthesize some new polycyclic compounds including chromene moiety and study their antimicrobial activity.

\section{MATERIALS AND METHODS}

Melting points were determined on the digital melting point apparatus (Electrothermal 9100, Electrothermal Engineering Ltd, serial No. 8694, Rochford, United Kingdom) and were uncorrected. IR data were measured on a Perkin-Elmer 1600 FTIR (Perkin-Elmer, USA) in $\mathrm{KBr}$ discs. ${ }^{1} \mathrm{H}$ and ${ }^{13} \mathrm{C}-\mathrm{NMR}$ spectra were recorded with a Bruker Avance spectrometer (300 and 75MHz) (Bruker, Germany) in DMSO- $\mathrm{d}_{6}$ and chemical shifts were determined in $\delta p p m$ to TMS as the standard internal solvent. Mass spectra (EI) were measured on
GCMS-QP 1000Ex70ev, Schimadz spectrometer, Japan (EI) at Micro Analytical Center, Cairo University, Egypt. Elemental analysis was measured by using a Perkin-Elmer microanalysis at $24^{\circ} \mathrm{C}$. The new derivatives were tested in vitro with respect to their antimicrobial activity at the Micro Analytical Center Faculty of Science, Cairo University. All reagents and solvents were of commercial grade.

Synthesis of 11-amino-12-(4-methoxyphenyl)-9-thioxo-9,12dihydro-8H-benzo [5,6]chromeno[2,3-b]pyridin-10-carbonitrile (2)

Cyanothioacetamide $(0.01 \mathrm{~mol})$ was added to a mixture of compound $\mathbf{1}(0.01 \mathrm{~mol})$ in ethanol $(30 \mathrm{ml})$ including a few drops of piperidine. The reaction mixture was allowed to reflux for $8 \mathrm{~h}$ then cooled down, after that transferred onto ice-cold water, and neutralized with $\mathrm{HCl}(10 \%)$. the filtration process was used to separate the resultant solid, which was cleaned and recrystallized from ethanol.

Synthesis of 11-imino-12-(4-methoxyphenyl)-10-phenyl, 10, 11 , dihydro-8H-benzo $[5,6]$ chromeno[2,3-b]pyrimidin-9-(12H) thione (3)

Phenyl isothiocyanate $(0.01 \mathrm{~mol})$ was added to the solution of compound $1(0.01 \mathrm{~mol})$ in dry dioxane $(20 \mathrm{ml})$ including $1 \mathrm{ml}$ pyridine for $10 \mathrm{~h}$. the reaction mixture was heated under reflux. The separated solid was formed after cooling at $25{ }^{\circ} \mathrm{C}$ and neutralized with $\mathrm{HCl}(10 \%)$. The precipitated product was filtered, washed with $\mathrm{H}_{2} \mathrm{O}$, dried and recrystallized from ethanol.

Synthesis of 9,11-diamino-12-(4-methoxyphenyl)-12H-benzo [5,6]chromeno[2,3-b]pyridin-10-carbonitrile (4)

A solution of compound $1(0.01 \mathrm{~mol})$ and malononitrile $(0.01 \mathrm{~mol})$ in absolute ethanol $(30 \mathrm{ml})$ containing a few drops of triethylamine 
was heated under reflux for $9 \mathrm{~h}$. The obtained solid was filtered and recrystallized from ethanol.

Synthesis of 12-(4-methoxyphenyl)-8,12-dihydro-9H-benzo [5,6]chromeno[2,3-d]pyrimidin-9,11(10H)-dithione (5)

A mixture of compound $\mathbf{1}(0.01 \mathrm{~mol})$ and an aqueous solution of 20 $\% \mathrm{KOH}$ in dimethyl sulfoxide $(20 \mathrm{ml})$ was stirred, and then carbon disulfide $(0.03 \mathrm{~mol})$ was added in several portions during $30 \mathrm{~min}$, after $1 \mathrm{~h}$ at $25^{\circ} \mathrm{C}$. The precipitated solid was separated, washed many times with $\mathrm{H}_{2} \mathrm{O}$, and recrystallized from dimethylformamide.

Synthesis of 2-(4,5-dihydro-1H-imidazol-2-yl)-1-(4-methoxyphenyl)-1H-benzo[f]-chromen-3-amine (6)

To a suspension of compound $1(0.01 \mathrm{~mol})$ in ethylenediamine ( 3 $\mathrm{ml}$ ), carbon disulfide $\mathrm{CS}_{2}(1 \mathrm{ml})$ was added dropwise and the reaction mixture was refluxed underwater bath control for $8 \mathrm{~h}$. After cooling, the deposited solid was filtered off, dried, and recrystallized from benzene.

Synthesis of 14-(4-methoxyphenyl)-5-phenyl-3,5,6,14-tetrahydro-2H-benzo[5,6]-chromeno[3,2-e]imidazo[1,2-c] pyrimidine (7)

A solution of compound $6(0.01 \mathrm{~mol})$ and benzaldehyde $(0.01 \mathrm{~mol})$ in absolute ethanol $(50 \mathrm{ml})$ containing $0.1 \mathrm{ml}$ of conc. $\mathrm{HCl}$ was refluxed for $14 \mathrm{~h}$. After cooling, the separated solid was collected and recrystallized from ethanol.

Synthesis of 14-(4-methoxyphenyl)-2,3,6,14-tetrahydro-5- $\mathrm{H}$ benzo[5,6]chromeno $[3,2-e]$ imidazo $[1,2-c]$ pyrimidin-5-thione (8)

A mixture of compound $6(0.01 \mathrm{~mol})$ and carbon disulfide $\mathrm{CS}_{2}$ (10 $\mathrm{ml})$ in dry pyridine $(25 \mathrm{ml})$ was refluxed under water-bath for $22 \mathrm{~h}$. After cooling, the reaction mixture was poured onto cold water. The formed solid was filtered off, dried and recrystallized from ethanol.

Synthesis of 14-(4-methoyphenyl)-3,14-dihydro-2H-benzo [5,6] chromeno[3,2-e] imidazo[1,2-c]pyrimidine (9)

A solution of compound $6(0.01 \mathrm{~mol})$, triethyl orthoformate $(30 \mathrm{ml})$ and acetic anhydride $(15 \mathrm{ml})$ were refluxed for $8 \mathrm{~h}$. The precipitate that obtained after cooling was filtered, dried and recrystallized from benzene.

Synthesis of 14-(4-methoxyphenyl)-2,3,6,14-tetrahydro-benzo $[5,6]$ chromeno[3,2-e]imidazo[1,2-c]pyrimidin-5-one (10)

A solution of $6(0.011 \mathrm{~mol})$ with ethylchroformate $(0.011 \mathrm{~mol})$ in dry pyridine $(30 \mathrm{ml})$ was refluxed for $1 \mathrm{~h}$. The separated solid whichformed was filtered, dried and recrystallized from dioxane.

Synthesis of 14-(4-methoxyphenyl)- $N$-phyenyl-2,3-dihydro$14 H$-benzo[5,6]-chromeno [3,2-e]imidazo[1,2-c] pyrimidin-5amine (11)

A solution of $6(0.011 \mathrm{~mol})$ and phenyl isothiocyanate $(0.011 \mathrm{ml})$ in dry pyridine $(10 \mathrm{ml})$ was heated under reflux for 7-8 h. After cooling, the precipitate was formed by pouring the mixture into cold water which filtered off, dried and recrystallized from DMF/ethanol.

Synthesis of 5-(chloromethyl)-14-(4-methoxyphenyl)-3,14dihydro-2H-benzo $[5,6]$-chromeno $[3,2-e]$ imidazo[1,2-c] pyrimidine (12)

A solution of compound $6(0.011 \mathrm{~mol})$ and chloroacetyl chloride $(0.011 \mathrm{~mol})$ in glacial acetic acid $(30 \mathrm{ml})$ was heated under reflux overwater bath for $15 \mathrm{~min}$. The precipitate that obtained by adding of aqueous sodium acetate was filtered off, dried and recrystallized from methanol.

Synthesis of 17-(4-methoxyphenyl)-2,3,5,6,7,8,16-octahydrobenzo[ $[5,6]$ chromeno $[3,2-e]$ imidazo $[1,2-a][1,5]$ diazocine $(13)$

A solution of compound $6(0.01 \mathrm{~mol})$ potassium carbonate anhydrous (3.03 g), and1, 4-dichlorobutane $(0.01 \mathrm{~mol})$ in dimethylformamide (20 $\mathrm{ml})$. The reaction mixture was stirred at $60^{\circ} \mathrm{C}$ for $9 \mathrm{~h}$. The reaction mixture was cooled down by adding cold water $(50 \mathrm{ml})$, and then titrated with $10 \%$ hydrochloric acid to reach pH7. The separated solid was collected by filtration, washed with distilled water and recrystallized from ethanol.

Diazotization of compound 1:Synthesis of 3-(chlorodiazenyl)1-(4-methoxyphenyl)-1H-benzo[f]chromen-2-carbonitrile (14)

To a suspended solution of compound $1(0.01 \mathrm{~mol})$ in conc. $\mathrm{HCl}$ (3 $\mathrm{ml})$, a solution of sodium nitrite $(0.01 \mathrm{~mol})$ in cold water $(2 \mathrm{ml})$ was added over $15 \mathrm{~min}$ at $0-5{ }^{\circ} \mathrm{C}$. The diazonium salt was used freshly in the next step.

\section{General procedure for the synthesis of compounds 15, 17}

The diazonium salt solution $14(0.005 \mathrm{~mol})$ was added slowly to a stirred solution of malononitrile and/or ethyl cyanoacetate $[0.005$ $\mathrm{mol})$ in acetone $(20 \mathrm{ml})$ at $0-5{ }^{\circ} \mathrm{C}$. The reaction was left overnight under stirring at room temperature. The separated product was then collected by filtration and washed with ethanol.

\section{(E)-3-(3,5-diamino-1H-pyrazol-4-y)diazenyl)-1-(4-methoxy-} phenyl)-1 $H$-benzo[f]chromeno-2-carbonitrile (16)

A solution of compound 15 (0.01 mol) and $\mathrm{NH}_{2} \mathrm{NH}_{2} 99 \%(5 \mathrm{ml})$ in ethanol $(20 \mathrm{ml})$ was refluxed for $5 \mathrm{~h}$. The excess solvent was evaporated under reduced pressure in vacuum. The separated solid was collected by filtration, air-dried and recrystallized from ethanol.

\section{3-(2,(3-Amino-5-oxo-1,5-dihydro-4H-pyrazol-4-ylidene)- hydrazinyl)-1H-benzo[f]chromene-2-carbonitrile (18)}

A mixture of compound 17 (0.01 mol) and $\mathrm{NH}_{2} \mathrm{NH}_{2} 99 \%(3 \mathrm{ml})$ in absolute ethanol $(20 \mathrm{ml})$ was refluxed for $5 \mathrm{~h}$. The precipitate that formed after cooling was filtered off, dried and recrystallized from absolute ethanol.

Synthesis of 1-(11-amino-12-(4-methoxyphenyl)-9-pyhenyl-12Hbenzo [5,6]-chromeno[2,3-b]pyridin-10-yl)ethan-1-one (19)

The reaction mixture of an equimolar amount of compound $\mathbf{1}(0.012$ $\mathrm{mol})$ and benzoyl acetone $(0.012 \mathrm{~mol})$ in ethanol $(20 \mathrm{ml})$ in the presence of sodium ethoxide was heated under reflux for $10 \mathrm{~h}$. The mixture was cooled down and then poured onto cold $\mathrm{H}_{2} \mathrm{O}$. The formed solid was collected by filtration and recrystallized from ethanol.

Synthesis of 2-amino14-(4-methoxyphenyl)-4-methy-5-phenyl$14 H$-benzo $[5,6]$ chromeno $[2,3 \mathrm{H}][1,6]$ naphthyridine-3carbonitrile (20).

A mixture of compound $19(0.012 \mathrm{~mol})$ and malononitrile $(0.012$ $\mathrm{mol})$ was allowed to heat under reflux in ethanol $(25 \mathrm{ml})$ including a few drops of piperidine as a catalyst for $16 \mathrm{~h}$. The separated solid that obtained after cooling was filtered off, washed with cold ethanol and recrystallized from DMF/ethanol.

\section{Antimicrobial evaluation}

Antibacterial and antifungal activity of the newly synthesized compounds was determined in vitro by the disc diffusion method [51] using a nutrient agar medium against the following microorganisms, Staphylococcus aureus (RCMB 29213), Bacillus subtilis (RCMB 010010) (Gram-positive bacteria), Escherichia coli (RCMB 27853), Pseudomonas aeuroginosa (RCMB 25922) (Gramnegative bacteria) and fungal species like Candida albicans (RCMB 05036), Asperigullusflavin (RCMB 02568). All strains were provided from the culture collection of the Regional Center for Mycology and Biotechnology (RCMB), Al-Azhar University, Cairo, Egypt. Ampicillin was the standard drug for antibacterial screening and amphotericin $B$ was used as a standard for antifungal screening.

\section{Method of testing}

The sterilized media was poured onto the sterilized Petri dishes (20 $\mathrm{ml}$, each Petri dish) and allowed to solidify. Wells of $6 \mathrm{~mm}$ diameter were made in the solidified media (Nutrient and MacConky agar media for bacteria and on Sabouraud dextrose agar for fungus.) with the help of sterile borer. A sterile swab was used to evenly distribute microbial suspension over the surface of solidified media and solutions of the tested samples ( $5 \mathrm{mg} / \mathrm{ml}$ ) were added to each well with the help of micropipette. The inhibition zones (IZ) of the test compounds were 
measured after $24-48 \mathrm{~h}$ incubation at $37{ }^{\circ} \mathrm{C}$ for bacteria and after $5 \mathrm{~d}$ incubation at $28{ }^{\circ} \mathrm{C}$ for fungi. The experiment was performed in triplicate and the average zone of inhibition was calculated.

\section{RESULTS AND DISCUSSION}

\section{Chemistry}

The pathway for the preparation of the target compounds is outlined under schemes 1-4. 2-Amino-1-(4-methoxyphenyl)-1 Hbenzo[f]chromene-3-carbonitrile (1) is the key compound in our study for the production of its functionalized derivatives [52]. Compound 1 was condensed with cyanothioacetamideunder basic condition to give11-amino-12-(4-methoxyphenyl)-9-thioxo-9,12dihydro-8H-benzo[5, 6] chromeno[2,3-b]pyridin-10-carbonitrile (2) (Scheme 1). The NMR spectra revealed two $\mathrm{D}_{2} \mathrm{O}$ replaceable main peaks at 6.9 and $8.3 \mathrm{ppm}$ related to the presence of $\mathrm{NH}_{2}$ and $\mathrm{NH}$ protons. Additionally, compound 1 was allowed to react with phenyl isothiocyanatein order to form 11-imino-12-(4-methoxyphenyl)-10phenyl,10,11-dihydro-8H-benzo[5, 6]chromeno[2,3-d] pyrimidin-9$(12 H)$ thione (3). The NMR spectra of compound 3 showed two $\mathrm{D}_{2} \mathrm{O}$ changeable singles at 8.3 and $8.4 \mathrm{ppm}$ corresponding to $\mathrm{NH}$ and $=\mathrm{NH}$, respectively. Basic catalyzed reaction of 1 with malononitrile afforded 9,11-diamino-12-(4-methoxyphenyl)-14,12 $H$-benzo $[5,6]$ chromeno[2,3- $b]$ pyridin-10-carbonitrile (4). Interaction of compound 1 with carbon disulfide in dimethyl sulfoxide gave 9mercapto [2,3- $d$ ] pyrimidin-11-thione derivative (5) (Scheme 1). The IR spectrum of compound 5 implied characteristic peak at $3100 \mathrm{~cm}^{-}$ ${ }^{1}$ which correlated to the NH group.

Furthermore, the reaction of 1 with ethylenediamine and a few drops of carbon disulfide ended up with 2-imidazolyl benzo [f]chromene derivative (6) (Scheme 2). The ${ }^{1} \mathrm{H}$ NMR spectrum of compound 6 indicated two $\mathrm{D}_{2} \mathrm{O}$ replaceable peaks at $\delta 8.2$ and 8.3 ppm corresponding to $\mathrm{NH}_{2}$ and $\mathrm{NH}$ protons, respectively.

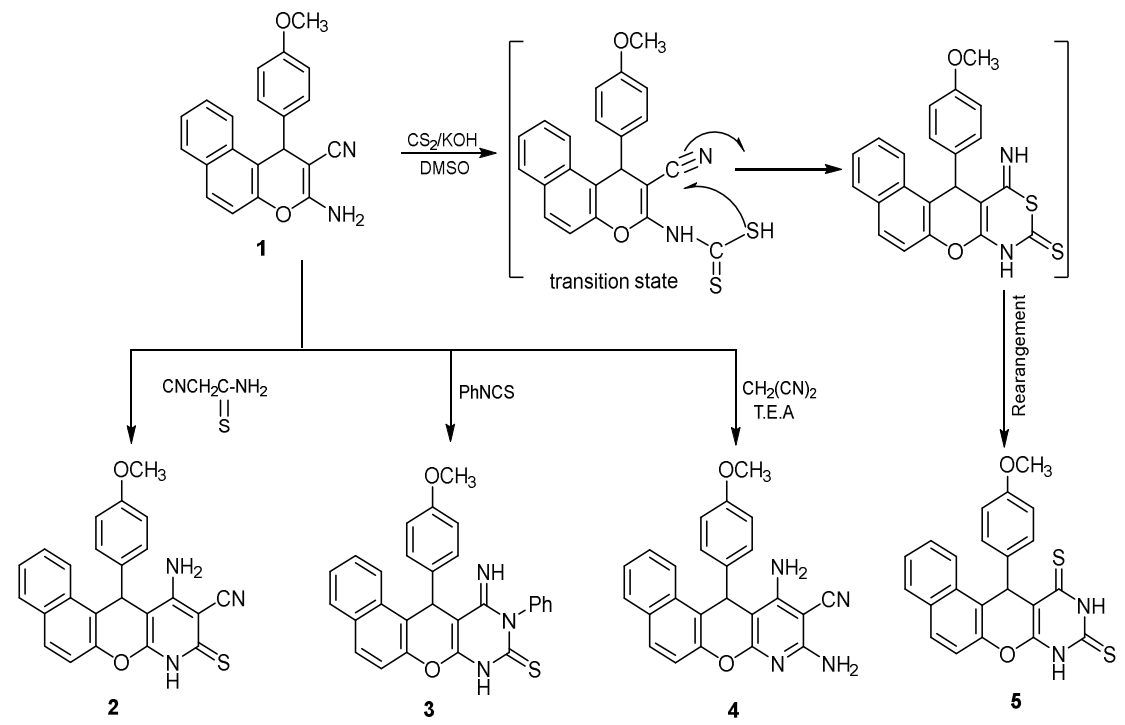

Scheme 1: Synthesis of benzo $[5,6]$ chromeno $[2,3-b]$ pyridine derivatives

Heating of compound 6 with benzaldehyde resulted in the corresponding 5-phenyl-benzochromenoimidazopyrimidine (7) as shown in Scheme 2. Moreover, benzochromeno-imidazopyridine-5thione (8) was achieved by reaction of compound $\mathbf{6}$ with carbon disulfide as described in Scheme 2 . The ${ }^{1} \mathrm{H}$ NMR spectrum of compound 8 showed signals at $\delta 6.8 \mathrm{ppm}$ and $\delta 8.3 \mathrm{ppm}$ attributed to pyridine- $\mathrm{C}_{5}$ proton and $\mathrm{NH}$ proton. Polycyclic systems were achieved through the reaction of compound $\mathbf{6}$ with triethyl orthoformate in acetic anhydride that led to the formation of benzochromeno-imidazopyridine (9).

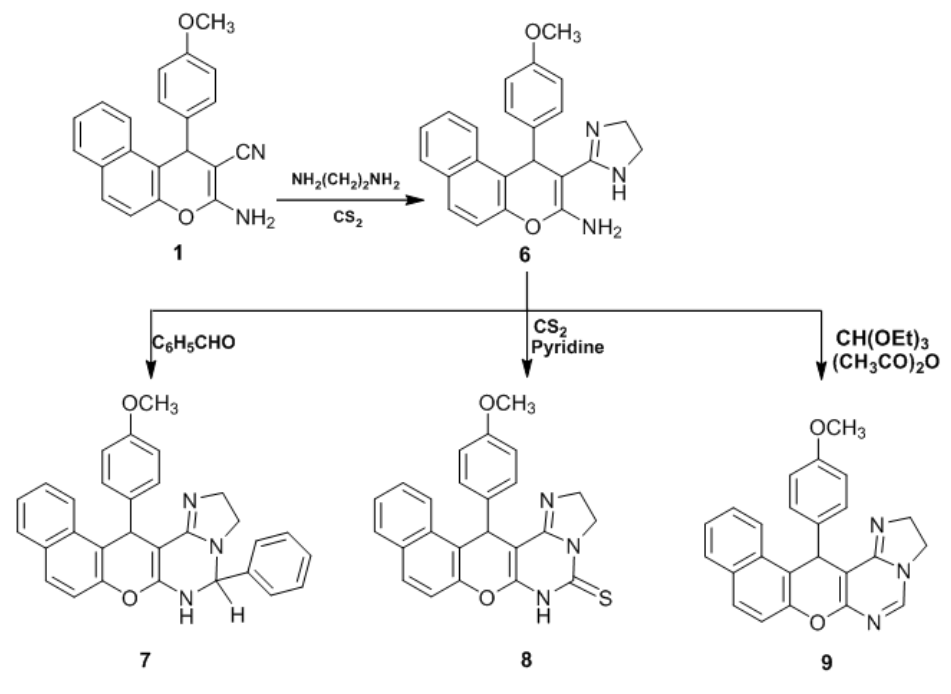

Scheme 2: Synthesis of benzo[5,6]-chromeno[3,2-e]imidazo[1,2-c]pyrimidine derivatives 
On the other hand, compound 6 was treated with ethyl chloroformate in pyridine to produce the imidazo[1,2-c]pyrimidin-5one derivative (10). Imidazo [1,2-c]pyrimidin-5-amine derivative (11) was obtained via intermolecular cyclization of the compound 6 with phenyl isothiocyanate. Compound 6 was heated with chloroacetyl chloride in glacial acetic acid at $60{ }^{\circ} \mathrm{C}$ to provide 5(chloromethyl)-14-(4-methoxyphenyl)-3,14-dihydro-2H-benzo [5,6] chromeno[3,2-e]imidazo[1,2-c]pyrimidine (12) (Scheme 3). IR data of compound 12 indicated a peak at $v 744.5 \mathrm{~cm}^{-1}$ for $\mathrm{C}-\mathrm{Cl}$ and disappearance of $\mathrm{NH}_{2}$ and $\mathrm{NH}$ groups that could be related to their involvement in the cyclization process. The mass spectrum of compound 12 showed a molecular ion peak at $m / z 472\left(\mathrm{M}^{+}, 2 \%\right)$, $406\left(\mathrm{M}^{+}+2,8 \%\right)$, which indicate the presence of $\mathrm{Cl}$ atom. The cyclo condensation of compound 6 with 1,4-dichlorobutane in DMF in the presence of anhydrous potassium carbonate led to the formation of imidazo[1,2-a][1,5]diazocine derivative (13).

Benzochromene 2-diazonium chloride 14 was achieved by diazotization of compound 1 through its treatment with cold $\mathrm{HCl}$ and $\mathrm{NaNO}_{2}$ aqueous solution. Coupling of compound 14 with active methylene compounds namely; malononitrile and ethylcyanoacetate led to the production of the hydrazono derivatives 15 and 17, respectively. Pyrazole derivatives 16 and 18 were created through the reaction of compounds 15 and/or 17 with hydrazine hydrate.

Treatment of compound 1 with benzoyl acetone in the presence of $\mathrm{C}_{2} \mathrm{H}_{5} \mathrm{ONa}$ led to the formation of 19 , which on turn reacted with malononitrile under the basic condition to produce benzo $[5,6]$ chromeno $[3,2,-h][1,6]$ naphtharidin-3-carbnitrile derivative (20) (Scheme 4).

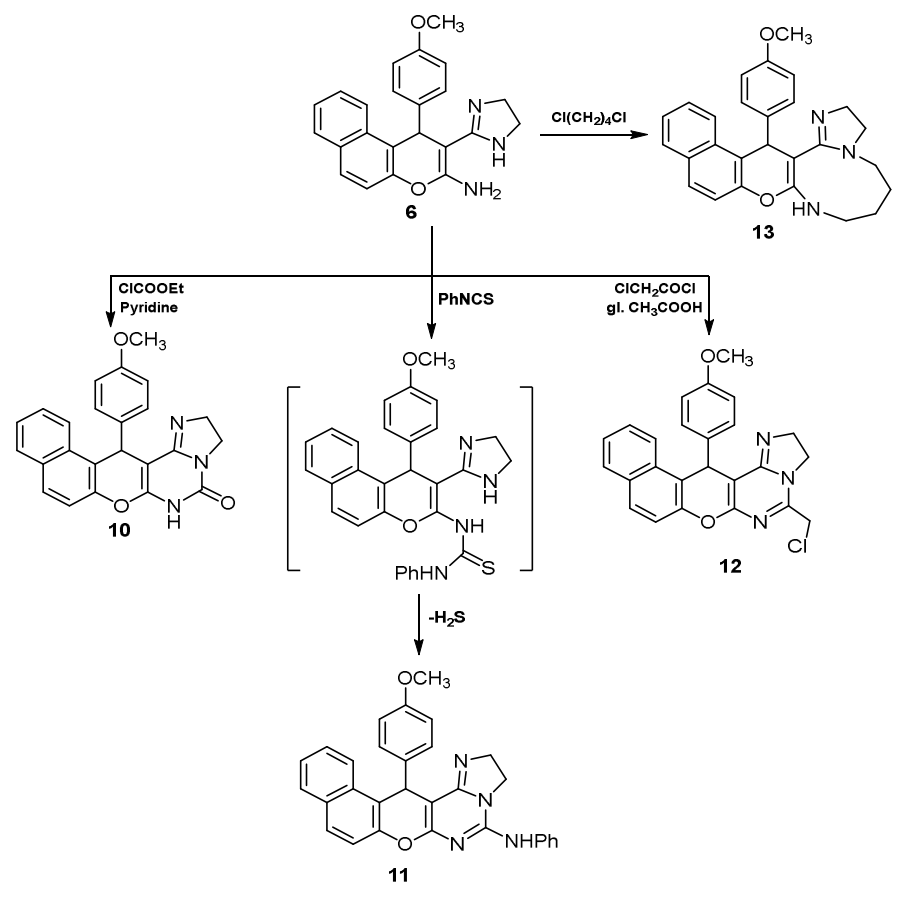

Scheme 3: Synthesis of benzo[5,6]chromeno[3,2-e]imidazo[1,2-c] pyrimidin-5-amine $(10,11,12)$ and 2,3,5,6,7,8,16-octahydrobenzo[5,6]chromeno[3,2-e]imidazo[1,2-a][1,5]diazocine (13)

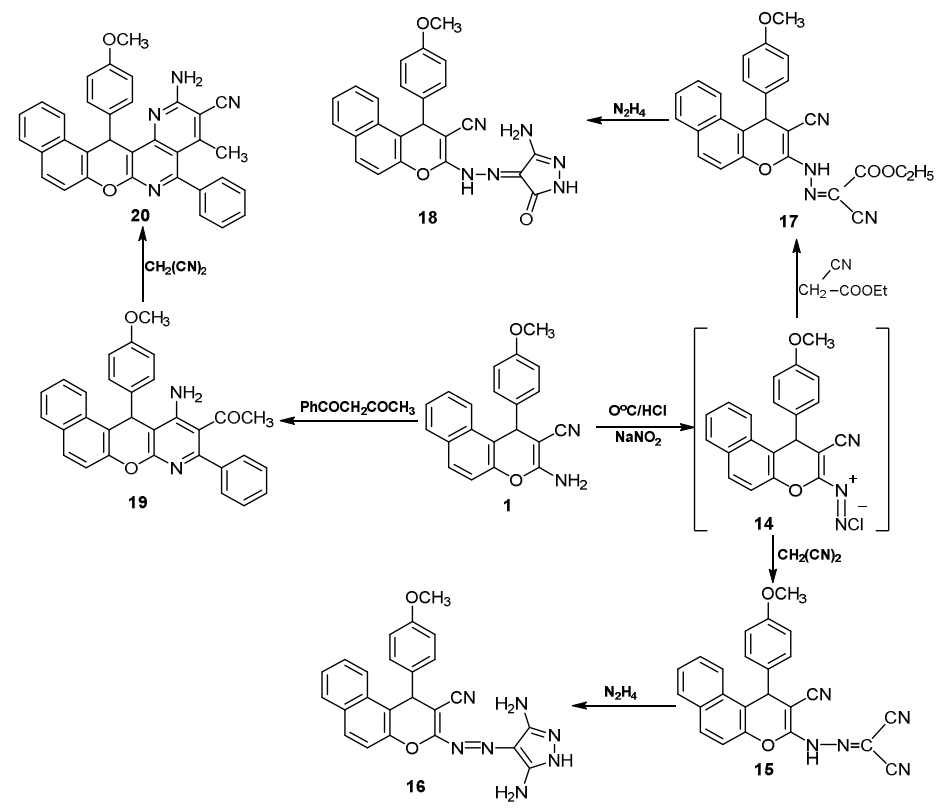

Scheme 4: Synthesis of benzo[5,6]chromene $(16,18)$, benzo[5,6]chromeno[2,3-b] pyridine (19) and benzo[5,6]chromeno[2,3H][1,6]naphthyridine $(20)$ 


\section{Spectral characterization of synthesized compounds}

11-Amino-12-(4-methoxyphenyl)-9-thioxo-9,12-dihydro-8Hbenzo[5,6]chromeno[2,3-b]pyridin-10-carbonitrile (2)

Black crystals; yield $83 \%$; mp $182{ }^{\circ} \mathrm{C}$; Anal Calcd (\%) for $\mathrm{C}_{24} \mathrm{H}_{17} \mathrm{~N}_{3} \mathrm{O}_{2} \mathrm{~S}$ (411.48): C, 70.05; H, 4.16; N, 10.21; found (\%) C, 70.1; H, 4.2; N; 10.1; IR ( $\left.\mathrm{KBr}, \mathrm{cm}^{-1}\right)$ : 3379, $3321\left(\mathrm{NH}, \mathrm{NH}_{2}\right) ; 3001$ for all (CH-aromatic); $2194(\mathrm{C} \equiv \mathrm{N}) ; 1562(\mathrm{C}=\mathrm{C}), 1273(\mathrm{C}=\mathrm{S}) ;{ }^{1} \mathrm{H}$ NMR (DMSO-d $\left.6, \delta \mathrm{ppm}\right): 3.8$ (s, $\left.3 \mathrm{H}, \mathrm{OCH}_{3}\right), 6.3\left(\mathrm{~s}, 1 \mathrm{H}\right.$, pyran-H), $6.9\left(\mathrm{~s}, 2 \mathrm{H}, \mathrm{NH}_{2}\right), 7.1-7.9(\mathrm{~m}, 10 \mathrm{H}$, $\mathrm{Ar}-\mathrm{H}), 8.3$ (s, 1H, NH); [13]C-NMR $\delta .26 .3,53.9,63.1,105.9,117.9$, 118.1, 118.8, 121.01, 123.6, 127.1, 128.2, 128.5, 128.7, 129.7, 129.8, $129.9,131.1,136.6,143.8,150.1,156.8,161.8$.

11-Imino-12-(4-methoxyphenyl)-10-phenyl,10,11,dihydro-8Hbenzo[5,6]chromeno[2,3-b]pyrimidin-9-(12H)thione (3)

Buff crystals; yield $62 \%$; mp $132{ }^{\circ} \mathrm{C}$; Anal Calcd (\%) for $\mathrm{C}_{28} \mathrm{H}_{21} \mathrm{~N}_{3} \mathrm{O}_{2} \mathrm{~S}$ (463.14): C, 72.55; H, 4.57; N, 9.06; found (\%) C, 72.1; H, 4.6; N, 9.1.; IR $\left(\mathrm{KBr}, \mathrm{cm}^{-1}\right): 3336,3309(\mathrm{NH}), 3001(\mathrm{CH}$-aromatic), $1689(\mathrm{C}=\mathrm{N})$, $1527(\mathrm{C}=\mathrm{C}), 1277(\mathrm{C}=\mathrm{S})$; ${ }^{1} \mathrm{H}$ NMR (DMSO-d $6, \delta \mathrm{ppm}$ ): $3.8(\mathrm{~s}, 3 \mathrm{H}$, $\left.\mathrm{OCH}_{3}\right), 6.3(\mathrm{~s}, \mathrm{H}$, pyran-H), 7.0-7.9 (m, 15H, $\mathrm{Ar}-\mathrm{H}), 8.3(\mathrm{~s}, 1 \mathrm{H}, \mathrm{NH}$, $\mathrm{D}_{2} \mathrm{O}$ exchangeable), $8.5\left(\mathrm{~s}, 1 \mathrm{H}, \mathrm{NH}, \mathrm{D}_{2} \mathrm{O}\right.$ exchangeable).

\section{9,11-Diamino-12-(4-methoxyphenyl)-12H-benzo[5,6]} chromeno[2,3-b]pyridin-10-carbonitrile (4)

White powder; yield $83 \%$; mp $142{ }^{\circ} \mathrm{C}$; Anal Calcd (\%) for $\mathrm{C}_{24} \mathrm{H}_{18} \mathrm{~N}_{4} \mathrm{O}_{2}$ (394.14): C, 73.68; H, 4.60; N, 14.20; found (\%) C, 73.1; H, 4.5; N, 14.1; IR (KBr, cm-1): 3390; $3288\left(\mathrm{NH}_{2}\right), 3011$ (CH-aromatic), 2200 $(\mathrm{CN}), 1610(\mathrm{C}=\mathrm{N}), 1530(\mathrm{C}=\mathrm{C}) ;{ }^{1} \mathrm{H}$ NMR (DMSO-d $\left.\mathrm{d}_{6}, \delta \mathrm{ppm}\right): 3.8(\mathrm{~s}$, $\left.3 \mathrm{H}, \mathrm{OCH}_{3}\right), 6.3(\mathrm{~s}, 1 \mathrm{H}$, pyran- $\mathrm{H}), 6.8\left(\mathrm{~s}, 2 \mathrm{H}, \mathrm{NH}_{2}, \mathrm{D}_{2} \mathrm{O}\right.$ exchangeable), $6.9\left(\mathrm{~s}, 2 \mathrm{H}, \mathrm{NH}_{2} . \mathrm{D}_{2} \mathrm{O}\right.$ exchangeable), 7.1-7.9 (m, 10H, Ar-H).

\section{2-(4-Methoxyphenyl)-8,12-dihydro-9H-benzo[5,6]chromeno} [2,3-d]pyrimidin-9,11(10H)-dithione (5)

White powder; yield $55 \%$; mp>300 ${ }^{\circ} \mathrm{C}$; Anal Calcd (\%) for $\mathrm{C}_{22} \mathrm{H}_{16} \mathrm{~N}_{2} \mathrm{O}_{2} \mathrm{~S}_{2}$ (404.07): C, 65.32; $\mathrm{H}, 3.99 ; \mathrm{N}, 6.93$; found (\%) C, 65.1; $\mathrm{H}, 3.5$; N, 6.7; IR (KBr, cm-1): $3100(\mathrm{NH}), 3012$ (CH-aromatic), 1609 $(\mathrm{C}=\mathrm{N}), 1531(\mathrm{C}=\mathrm{C}) ;{ }^{1} \mathrm{H}$ NMR (DMSO-d 6 , $\left.\delta \mathrm{ppm}\right): 3.8\left(\mathrm{~s}, 3 \mathrm{H}, \mathrm{OCH}_{3}\right), 6.3$ $\left(\mathrm{s}, 1 \mathrm{H}\right.$, pyran-H) $7.01-7.8(\mathrm{~m}, 10 \mathrm{H}, \mathrm{Ar}-\mathrm{H}), 8.1\left(\mathrm{~s}, 1 \mathrm{H}, \mathrm{NH}, \mathrm{D}_{2} \mathrm{O}\right.$, exchangeable), 8.3 (s, $1 \mathrm{H}, \mathrm{NH}, \mathrm{D}_{2} \mathrm{O}$ exchangeable).

\section{2-(4,5-Dihydro-1H-imidazol-2-yl)-1-(4-methoxyphenyl)-1H- benzo[f]-chromen-3-amine (6)}

Black brown powder; yield $65 \%$; $\mathrm{mp} 152{ }^{\circ} \mathrm{C}$; Anal Calcd (\%) for $\mathrm{C}_{23} \mathrm{H}_{21} \mathrm{~N}_{3} \mathrm{O}_{2}$ (371.16): C, 74.37; H, 5.70; N, 11.31; found (\%): C, 74.1; $\mathrm{H}, 5.6$; N, 11.1; IR $\left(\mathrm{KBr}, \mathrm{cm}^{-1}\right): 3360,33.48\left(\mathrm{NH}, \mathrm{NH}_{2}\right), 3002(\mathrm{CH}-$ aromatic), $1604(\mathrm{C}=\mathrm{N}), 1573(\mathrm{C}=\mathrm{C}) ;\left({ }^{1} \mathrm{H}\right.$ NMR $\left.-\mathrm{d}_{6}, \delta \mathrm{ppm}\right): 3.8(\mathrm{~s}, 3 \mathrm{H}$, $\left.\mathrm{OCH}_{3}\right) 6.3\left(\mathrm{~s}, 1 \mathrm{H}\right.$, pyran-H) 6.55-6.57 (m, $2 \mathrm{H}$, imidazolidine- $\left.\mathrm{C}_{4}-\mathrm{H}\right)$, 6.80-7.00 (m, $2 \mathrm{H}$, imidazolidine- $\left.\mathrm{C}_{5}-\mathrm{H}\right)$, 7.1-7.9 (m, 10H, Ar- $\left.\mathrm{H}\right), 8.2$ $\left(\mathrm{s}, \quad 2 \mathrm{H}, \quad \mathrm{NH}_{2}, \quad \mathrm{D}_{2} \mathrm{O}\right.$ exchangeable); $8.3 \quad\left(\mathrm{~s}, \quad 1 \mathrm{H}, \quad \mathrm{NH}, \quad \mathrm{D}_{2} \mathrm{O}\right.$ exchangeable);[13]C, NMR (DMSO-d $6, \delta$ ppm):, 21.9, 55.6, 99.5, 113.2, 118.9, 119.2, 120.6, 121.9, 122.1, 122.5, 123.2, 123.7, 125.4, $126.3,127.9,128.3,143.5,158.6,159.1,160.1,160.2$.

\section{4-(4-Methoxyphenyl)-5-phenyl-3,5,6,14-tetrahydro-2 $\mathrm{H}$ - benzo $[5,6]$ chromeno $[3,2-e]$ imidazo $[1,2-c]$ pyrimidine (7)}

White crystal; yield $82 \%$; mp>300 ${ }^{\circ} \mathrm{C}$; Anal Calcd (\%) for $\mathrm{C}_{30} \mathrm{H}_{25} \mathrm{~N}_{3} \mathrm{O}_{2}$ (459.54): C, 78.41; H, 5.48; N, 9.14; found (\%): C, 78.1; H, 5.3; N, 9.2. IR $\left(\mathrm{KBr}, \mathrm{cm}^{-1}\right): 3320(\mathrm{NH}) ; 3005$, (CH-aromatic); $1561(\mathrm{C}=\mathrm{N}) ; 1510$ $(\mathrm{C}=\mathrm{C}) ;{ }^{1} \mathrm{H}$ NMR (DMSO-d $\left.6, \delta \mathrm{ppm}\right) ; 3.1\left(\mathrm{~s}, 3 \mathrm{H}, \mathrm{OCH}_{3}\right), 3.6(\mathrm{~m}, 2 \mathrm{H}$, imidazo- $\left.\mathrm{C}_{2}-\mathrm{H}\right), 3.8\left(\mathrm{~m}, 2 \mathrm{H}\right.$, imidazo- $\left.\mathrm{C}_{3}-\mathrm{H}\right), 6.3(\mathrm{~s}, 1 \mathrm{H}$, pyran- $\mathrm{H}), 6.8(\mathrm{~s}$, $\mathrm{H}$, pyrimidine- $\mathrm{H}), 7.04-7.5(\mathrm{~m}, 15 \mathrm{H}, \mathrm{Ar}-\mathrm{H}), 8.5\left(\mathrm{~s}, \mathrm{H}, \mathrm{NH}, \mathrm{D}_{2} \mathrm{O}\right.$ exchangeable).

\section{4-(4-Methoxyphenyl)-2,3,6,14-tetrahydro-5- $H$-benzo} [5,6]chromeno[3,2-e]imidazo [1,2-c]pyrimidin-5-thione (8)

Brown powder; yield $70 \%$; mp $222{ }^{\circ} \mathrm{C}$; Anal Calcd (\%) for $\mathrm{C}_{24} \mathrm{H}_{19} \mathrm{~N}_{3} \mathrm{O}_{2} \mathrm{~S}$ (413): C, 68.7; M, 4.6; N, 10.1; found (\%): C, 69.6; H, 4.5; N, 10.2.; IR $\left(\mathrm{KBr}, \mathrm{cm}^{-1}\right): 3241(\mathrm{NH}) ; 3000$ (CH-aromatic); $1650(\mathrm{C}=\mathrm{N}) ;(\mathrm{C}=\mathrm{C}), 1447$ $(\mathrm{C}=\mathrm{S}) ;{ }^{1} \mathrm{H}$ NMR (DMSO-d $\left.6, \delta \mathrm{ppm}\right): 3.1\left(\mathrm{~s}, 3 \mathrm{H}, \mathrm{OCH}_{3}\right), 3.6(\mathrm{~m}, 2 \mathrm{H}$, imidazo- $\left.\mathrm{C}_{2}-\mathrm{H}\right), 3.8\left(\mathrm{~m}, 2 \mathrm{H}\right.$, imidazo- $\left.\mathrm{C}_{3}-\mathrm{H}\right), 6.3(\mathrm{~s}, 1 \mathrm{H}$, pyran- $\mathrm{H}), 7.05-$ $7.9(\mathrm{~m}, 10 \mathrm{H}, \mathrm{Ar}-\mathrm{H}), 8.4\left(\mathrm{~s}, 1 \mathrm{H}, \mathrm{NH}, \mathrm{D}_{2} \mathrm{O}\right.$ exchangeable).
14-(4-Methoyphenyl)-3,14-dihydro-2 $H$-benzo[5,6]chromeno $[3,2-e]$ imidazo $[1,2-c]$ pyrimidine $(9)$

Brown powder; yield $75 \%$ : mp $252{ }^{\circ} \mathrm{C}$; Anal Calcd (\%) for $\mathrm{C}_{24} \mathrm{H}_{19} \mathrm{~N}_{3} \mathrm{O}_{2}$ (381.15): C, 75.57; $\mathrm{H}, 5.02 ; \mathrm{N}, 11.02$; found (\%): C, 75.1; H, 5.1; N, 11.1; IR (KBr, cm$\left.{ }^{-1}\right): 3008$ (CH-aromatic); $1601(\mathrm{C}=\mathrm{N})$, $1573(\mathrm{C}=\mathrm{C}) ;\left({ }^{1} \mathrm{H}\right.$ NMR- $\left.\mathrm{d}_{6}, \delta \mathrm{ppm}\right): 3.1\left(\mathrm{~s}, 3 \mathrm{H}, \mathrm{OCH}_{3}\right), 3.6(\mathrm{~m}, 2 \mathrm{H}$, imidazo- $\left.\mathrm{C}_{2} \mathrm{H}\right), 3.8\left(\mathrm{~m}, 2 \mathrm{H}\right.$, imidazo- $\left.\mathrm{C}_{3}-\mathrm{H}\right), 6.1(\mathrm{~s}, 1 \mathrm{H}$, pyran- $\mathrm{H}), 6.9(\mathrm{~s}$, $\mathrm{H}$, pyrimidine-H), 7.11-7.58 (m, 10H, Ar-H);[13]C NMR: $\delta 35.1,46.1$, $55.1,57.2,109.7,117.2,117.3,119.8,120.8,120.9,121.6,121.8$, 128.7, 129.4, 129.8, 129.9, 130.1, 132.2, 135.1, 135.7, 137.7, 138.8, $140.1,140.2$.

\section{4-(4-Methoxyphenyl)-2,3,6,14-tetrahydro-benzo[5,6] chromeno[3,2-e]imidazo[1,2-c]pyrimidin-5-one (10)}

White crystals; yield $65 \%$; mp $>300{ }^{\circ} \mathrm{C}$; Anal Calcd (\%): for $\mathrm{C}_{24} \mathrm{H}_{19} \mathrm{~N}_{3} \mathrm{O}_{3}$ (397.14): C, 72.53; H, 4.82; N, 10.57; found (\%): C, 72.4; $\mathrm{H}, 4.7$; N, 10.4; IR (KBr, cm$\left.{ }^{-1}\right): 3007$ (CH-aromatic), $1602(\mathrm{C}=0)$, $1553(\mathrm{C}=\mathrm{C}) ;{ }^{1} \mathrm{H}$ NMR (DMSO-d $\left.6, \delta \mathrm{ppm}\right): 3.8\left(\mathrm{~s}, 3 \mathrm{H}, \mathrm{OCH}_{3}\right), 6.1(\mathrm{~s}, \mathrm{H}$, pyran- $\mathrm{H}), 5.4\left(\mathrm{~m}, 2 \mathrm{H}\right.$, imidazo- $\left.\mathrm{C}_{2}-\mathrm{H}\right), 6.1\left(\mathrm{~m}, 2 \mathrm{H}\right.$, imidazo- $\left.\mathrm{C}_{3}-\mathrm{H}\right)$, 7.01-7.6 (m, 10H, Ar-H) 8.3 (s, H, NH, $\mathrm{D}_{2} \mathrm{O}$ exchangeable).

14-(4-Methoxyphenyl)- $N$-phyenyl-2,3-dihydro-14H-benzo [5,6]chromeno[3,2-e] imidazo[1,2-c] pyrimidin-5-amine (11)

Brown crystals; yield $65 \%$ mp $287{ }^{\circ} \mathrm{C}$; Anal Calcd (\%) for $\mathrm{C}_{30} \mathrm{H}_{24} \mathrm{~N}_{4} \mathrm{O}_{2}$ (472.19): C, 76.25; H, 5.12; N, 11.80; found (\%). C, 76.1; H, 5.1; N, 11.7; IR (KBr, cm-1): 3320 (NH), 3007 (CH-aromatic), 1602 $(\mathrm{C}=\mathrm{N}), 1510(\mathrm{C}=\mathrm{C}) ;{ }^{1} \mathrm{H}$ NMR $\left(\mathrm{DMSO}_{-} \mathrm{d}_{6}, \delta \mathrm{ppm}\right): 3.8\left(\mathrm{~s}, 3 \mathrm{H}, \mathrm{OCH}_{3}\right), 6.1$ $(\mathrm{s}, 1 \mathrm{H}$, pyran- $\mathrm{H}), 5.3\left(\mathrm{~m}, 2 \mathrm{H}\right.$, imidazo- $\left.\mathrm{C}_{2}-\mathrm{H}\right), 6.5\left(\mathrm{~m}, 2 \mathrm{H}\right.$, imidazo $\mathrm{C}_{3}-$ H), 7.1-7.9 (m, 15H, Ar-H), $8.3\left(\mathrm{~s}, \mathrm{H}, \mathrm{NH}, \mathrm{D}_{2} \mathrm{O}\right.$ exchangeable);[13]C (DMSO- $\mathrm{d}_{6}, \delta \mathrm{ppm}$ ): $36.2,56.0,57.6,109.8,117.1,117.2,119.7,120.9$, 121.0, 123.6, 123.6, 125.3, 127.5, 128.8, 129.4,129.5, 129.6, 129.8, $129.9,130.1,132.2,142.7,142.8,147.1,160.1,162.1$

\section{5-(Chloromethyl)-14-(4-methoxyphenyl)-3,14-dihydro-2H-} benzo[5,6]chromeno $[3,2-e]$ imidazo[1,2-c]pyrimidine (12)

Brown crystal; yield $60 \%$; mp $>300{ }^{\circ} \mathrm{C}$; Anal Calcd (\%) for $\mathrm{C}_{25} \mathrm{H}_{20} \mathrm{ClN}_{3} \mathrm{O}_{2}$ (429.90) C; 69.85; H, 4.69; N, 9.77; found (\%): C; 69.7; $\mathrm{H}, 4.5$; N, 9.6; IR $\left(\mathrm{KBr}, \mathrm{cm}^{-1}\right)$ : 3001 (CH-aromatic), $1601(\mathrm{C}=\mathrm{N}), 1510$ $(\mathrm{C}=\mathrm{C}), 745(\mathrm{C}-\mathrm{Cl}){ }^{1}{ }^{1} \mathrm{H}$ NMR $\left(\mathrm{DMSO}-\mathrm{d}_{6}, \delta \mathrm{ppm}\right): 3.1\left(\mathrm{~s}, 3 \mathrm{H}, \mathrm{OCH}_{3}, 3.6\right.$ $\left(\mathrm{m}, 2 \mathrm{H}\right.$, imidazo- $\left.\mathrm{C}_{2}-\mathrm{H}\right), 3.8\left(\mathrm{~m}, 2 \mathrm{H}\right.$, imidazole $\left.-\mathrm{C}_{3}-\mathrm{H}\right), 6.3(\mathrm{~s}, 1 \mathrm{H}$, pyran-H), $5.1\left(\mathrm{~s}, 2 \mathrm{H}, \mathrm{CH}_{2}-\mathrm{Cl}\right), 7.1-7.9(\mathrm{~m}, 10 \mathrm{H}, \mathrm{Ar}-\mathrm{H})$

17-(4-methoxyphenyl)-2,3,5,6,7,8,16-octahydro-benzo
[5,6]chromeno[3,2-e]imidazo[1,2-a][1,5]diazocine (13)

Brown crystals; yield $83 \%$; mp $177{ }^{\circ} \mathrm{C}$; Anal Calcd (\%) for $\mathrm{C}_{27} \mathrm{H}_{27} \mathrm{~N}_{3} \mathrm{O}_{2}$ (425.21): C, 76.19; $\mathrm{H}, 6.3 ; \mathrm{N}, 9.8$; found (\%): C, 76.1; H, 9.2; N, 9.1; IR (KBr, cm ${ }^{-1} 3332(\mathrm{NH}) ; 3005$ (CH-aromatic); $1651(\mathrm{C}=\mathrm{N}), 1521(\mathrm{C}=\mathrm{C}) ;{ }^{1} \mathrm{H}$ NMR (DMSO-d $\left.6, \delta \mathrm{ppm}\right): 3.1(\mathrm{~m} \mathrm{2H}, \mathrm{C}-$ $\left.{ }_{6}-\mathrm{H}\right), 3.3\left(\mathrm{~m}, 2 \mathrm{H}, \mathrm{C}_{7}-\mathrm{H}\right), 3.8\left(\mathrm{~s}, 3 \mathrm{H}, \mathrm{OCH}_{3}\right), 4.6\left(\mathrm{~m}, 2 \mathrm{H}, \mathrm{C}_{5}-\mathrm{H}\right), 5.1$ (s, H, pyran- $\mathrm{H}), 5.3\left(\mathrm{~m}, 2 \mathrm{H}\right.$, imidazo- $\left.\mathrm{C}_{2}-\mathrm{H}\right), 6.5(\mathrm{~m}, 2 \mathrm{H}$, imidazo$\left.\mathrm{C}_{3}-\mathrm{H}\right)$ 7.17-7.91 (m, 10H, Ar-H), $8.1 \quad\left(\mathrm{~s}, \quad \mathrm{H}, \quad \mathrm{NH}, \mathrm{D}_{2} \mathrm{O}\right.$ exchangeable).

$N$-(2-Cyano-1-(4-methoxyphenyl)-1H-benzo[f]chromen-3-yl) carbonohydrazonoyldicyanide (15)

Brown crystals; yield $75 \%$; mp $132{ }^{\circ} \mathrm{C}$; Anal Calcd (\%) for $\mathrm{C}_{24} \mathrm{H}_{15} \mathrm{~N}_{5} \mathrm{O}_{2}$ (405.12): C, 71.10; $\mathrm{H}, 3.73 ; \mathrm{N}, 17.27$; found (\%): C, 71.2; H, 3.5; N, 17.1; IR ( $\left.\mathrm{KBr}, \mathrm{cm}^{-1}\right) 3332(\mathrm{NH}), 3001$ (CH-aromatic), 2198 $(\mathrm{C} \equiv \mathrm{N}) ; 1643(\mathrm{C}=\mathrm{N}) 1504(\mathrm{C}=\mathrm{C}) ;{ }^{1} \mathrm{H}$ NMR (DMSO-d $\left.{ }_{6} \delta \mathrm{ppm}\right): 3.8(\mathrm{~s}, 3 \mathrm{H}$, $\left.\mathrm{OCH}_{3}\right), 4.3(\mathrm{~s}, 1 \mathrm{H}$, pyran-H), 7.1-7.9 (m, $10 \mathrm{H}, \mathrm{Ar}-\mathrm{H}), 8.3\left(\mathrm{~s}, \mathrm{H}, \mathrm{NH}, \mathrm{D}_{2} \mathrm{O}\right.$ exchangeable).

(E)-3-(3,5-Diamino-1H-pyrazol-4-y)diazenyl)-1-(4methoxyphenyl)-1H-benzo[f]chromeno-2-carbonitrile (16)

Brown powder; yield $57 \%$; mp $212{ }^{\circ} \mathrm{C}$; Anal Calcd (\%) for $\mathrm{C}_{24} \mathrm{H}_{19} \mathrm{~N}_{7} \mathrm{O}_{2}$ (437.16): C, 65.89; $\mathrm{H}, 4.38 ; \mathrm{N}, 22.41$; found (\%): C, 65.7; H, 4.1; N, 22.3; IR (KBr, cm-1): 3387, 3336, $3208\left(\mathrm{NH}, \mathrm{NH}_{2}\right) 2187$ $(\mathrm{CN}), 1651(\mathrm{C}=\mathrm{N}), 1562(\mathrm{C}=\mathrm{C}), 1408(\mathrm{~N}=\mathrm{N}) ;{ }^{1} \mathrm{H}$ NMR (DMSO-d $6, \delta$ ppm): $3.8\left(\mathrm{~s}, 3 \mathrm{H}, \mathrm{OCH}_{3}\right), 6.1(\mathrm{~s}, 1 \mathrm{H}$, pyran-H), $6.9(\mathrm{~s}, 1 \mathrm{H}, \mathrm{NH}$, pyrazole- $\mathrm{NH}, \mathrm{D}_{2} \mathrm{O}$ exchangeable), 7.1-7.8 $(\mathrm{m}, 10 \mathrm{H}, \mathrm{Ar}-\mathrm{H}), 8.4(\mathrm{~s}, 4 \mathrm{H}$, $2 \mathrm{NH}_{2}$, pyrazole, $\mathrm{D}_{2} \mathrm{O}$ exchangeable). 
(Z) Ethyl-2-cyano-2-(2-(2-cyano-1-(4-methoxyphenyl)-1H-benzo [f] chromene-3-yl) hydrazono) acetate (17)

Brown crystals; yield $69 \%$; mp $122^{\circ} \mathrm{C}$; Anal Calcd (\%)| for $\mathrm{C}_{26} \mathrm{H}_{20} \mathrm{~N}_{4} \mathrm{O}_{4}$ (452.46): C, 69; H, 4.4; N, 12.3; found (\%): C, 68.1; H, 4.3; N, 12.3; IR (KBr, cm${ }^{-1}$ ): 3357 (NH): 3008 (CH-aromatic), $2194(\mathrm{CN}) ; 1698$ (C=0), $1612(\mathrm{C}=\mathrm{N}), 1573(\mathrm{C}=\mathrm{C}) ;{ }^{1} \mathrm{H}$ NMR (DMSO- $\left.\mathrm{d}_{6}, \delta \mathrm{ppm}\right): 1.2\left(\mathrm{t}, 3 \mathrm{H}, \mathrm{CH}_{3}, \mathrm{~J}=\right.$ $7.2 \mathrm{~Hz}), 4.6\left(\mathrm{q}, 2 \mathrm{H}, \mathrm{CH}_{2}, \mathrm{~J}=7.2 \mathrm{~Hz}\right), 3.8\left(\mathrm{~s}, 3 \mathrm{H}, \mathrm{OCH}_{3}\right), 6.1(\mathrm{~s}, 1 \mathrm{H}$, pyran$\mathrm{H})$, 7.05-7.8 (m, 10H, Ar-H), 8.6 (s, H, NH, $\mathrm{D}_{2} \mathrm{O}$ exchangeable).

\section{3-(2,(3-Amino-5-oxo-1,5-dihydro-4H-pyrazol-4-ylidene)-} hydrazinyl)-1 $H$-benzo[f]chromene-2-carbonitrile (18)

Brown crystal; yield $65 \%$; mp $232{ }^{\circ} \mathrm{C}$; Anal Calcd (\%) for $\mathrm{C}_{24} \mathrm{H}_{18} \mathrm{~N}_{6} \mathrm{O}_{3}$ (438.14): C; 65.75; $\mathrm{H}, 4.14 ; \mathrm{N}, 19.17$; found (\%): C; 65.6; H, 4; N, 19.2; IR (KBr, cm-1): 3325, 3209 (NH, NH $\left.{ }_{2}\right), 2197$ (CN), 1680 $(\mathrm{C}=0), 1602(\mathrm{C}=\mathrm{N}), 1581(\mathrm{C}=\mathrm{C}) ;{ }^{1} \mathrm{H}$ NMR $\left(\mathrm{DMSO}^{-} \mathrm{d}_{6}, \delta \mathrm{ppm}\right): 3.8(\mathrm{~s}$, $\left.3 \mathrm{H}, \mathrm{OCH}_{3}\right), 6.01(\mathrm{~s}, 1 \mathrm{H}$, pyran-H) 7.01-7.6 (m, 10H, Ar-H), $8.1(\mathrm{~s}, 2 \mathrm{H}$, pyrazole- $\mathrm{C}_{3}-\mathrm{NH}_{2}, \quad \mathrm{D}_{2} \mathrm{O}$ exchangeable $) ; 8.2(\mathrm{~s}, 1 \mathrm{H}$, pyrazole- $\mathrm{NH}$, $\mathrm{D}_{2}$ Oexchangeable), 8.3 (s, 1H, $\mathrm{C}_{3}-\mathrm{NH}, \mathrm{D}_{2} \mathrm{O}$ exchangeable).

1-(11-Amino-12-(4-methoxyphenyl)-9-pyhenyl-12Hbenzo[5,6]chromeno[2,3-b]pyridin-10-yl)ethan-1-one (19)

Pale bag powder; yield $45 \%$; mp $122{ }^{\circ} \mathrm{C}$; Anal Calcd (\%) for $\mathrm{C}_{31} \mathrm{H}_{24} \mathrm{~N}_{2} \mathrm{O}_{3}$ (472.18): C, 78.78; $\mathrm{H}, 5.08 ; \mathrm{N}, 5.92$; found (\%): C, 78.3; $\mathrm{H}$,
5.4; N, 5.8; IR (KBr, cm-1): $3325\left(\mathrm{NH}_{2}\right), 3001$ (CH-aromatic), 1712 $(\mathrm{C}=0), 1666(\mathrm{C}=\mathrm{N}), 1563(\mathrm{C}=\mathrm{C}){ }^{1}{ }^{1} \mathrm{H}$ NMR $\left(\mathrm{DMSO}-\mathrm{d}_{6}, \delta \mathrm{ppm}\right): 3.3(\mathrm{~s}$, $\left.3 \mathrm{H}, \mathrm{COCH}_{3}\right), 3.8\left(\mathrm{~s}, 3 \mathrm{H}, \mathrm{OCH}_{3}\right), 6.1(\mathrm{~s}, 1 \mathrm{H}$, pyran-H), 7.1-7.8 $(\mathrm{m}, 15 \mathrm{H}$, $\mathrm{Ar}-\mathrm{H}), 8.6\left(\mathrm{~s}, 2 \mathrm{H}, \mathrm{NH}_{2}, \mathrm{D}_{2} \mathrm{O}\right.$ exchangeable).

\section{2-Amino14-(4-methoxyphenyl)-4-methy-5-phenyl-14H-benzo [5,6]chromeno-[2,3H][1,6]naphthyridine-3-carbonitrile (20)}

Brown powder; yield $82 \%$; mp $>300^{\circ} \mathrm{C}$; Anal Calcd (\%) for $\mathrm{C}_{34} \mathrm{H}_{24} \mathrm{~N}_{4} \mathrm{O}_{2}$ (520.19): C, 78.44; H, 4.65; N, 10.76; found (\%): C, 78.3; H, 4.5; N, 10.6; IR (KBr, cm$\left.{ }^{-1}\right): 3320\left(\mathrm{NH}_{2}\right), 3002$ (CH-aromatic), $2212(\mathrm{CN}), 1656(\mathrm{C}=\mathrm{N})$, 1560 (C=C); ${ }^{1} \mathrm{H}$ NMR(DMSO-d 6 , $\delta$ ppm) $3.02\left(\mathrm{~s}, 3 \mathrm{H}, \mathrm{CH}_{3}\right), 3.8(\mathrm{~s}, 3 \mathrm{H}$, $\left.\mathrm{OCH}_{3}\right), 6.3\left(\mathrm{~s}, 1 \mathrm{H}\right.$, pyran-H), 7.1-7.9 (m, 15H, Ar-H), 8.3(s, $\left.2 \mathrm{H}, \mathrm{NH}_{2}\right) ;[13] \mathrm{C}$ NMR: $\delta$ 23.1, 55.1, 63.2, 117.1, 118.1, 119.1, 123.2, 125.3, 128.1, 128.8 $28.9,130.1,130.2,130.6,130.7,130.9,140.1,140.2,140.5,140.6,140.7$, $140.8,140.9,141.2,147.1,150.3,151.1,152.1,153.1,160.1$.

\section{Antimicrobial activity}

The screening results are tabulated in table 1 . Among all the tested compounds, 1-3, 5 and 8 implied their optimum antibacterial activity while the compounds 4, 6 and 9-13, 15 showed a moderate to weak antibacterial activity against B. subtilis, $S$. aureus, E. coli and $P$. aeruginesa. In contrast, the compounds $1,6,11,15$ are highly active against $A$. flavin and $C$. albicans while the other compounds exhibit a moderate to weak activity.

Table 1: Antimicrobial activity of most active compounds

\begin{tabular}{|c|c|c|c|c|c|c|}
\hline \multicolumn{7}{|c|}{ Antimicrobial activity of most active compounds } \\
\hline Inhibition zon & mm) \pm Standard de & $n^{a}$ & & & & \\
\hline \multirow[t]{2}{*}{$\begin{array}{l}\text { Compd. } \\
\text { No. }\end{array}$} & $\begin{array}{l}\text { B. subtilis(RCMB } \\
010010 \text { ) }\end{array}$ & $\begin{array}{l}\text { S. aureus } \\
\text { (RCMB 29213) }\end{array}$ & $\begin{array}{l}\text { E. coli (RCMB } \\
\text { 27853) } \\
\end{array}$ & $\begin{array}{l}\text { P. aeruginosa } \\
\text { (RCMB 25922) }\end{array}$ & $\begin{array}{l}\text { C. albicans } \\
\text { (RCMB 05036) }\end{array}$ & $\begin{array}{l}\text { A. fumigatus } \\
\text { (RCMB 02568) }\end{array}$ \\
\hline & \multicolumn{6}{|c|}{ Test compounds $5 \mathrm{mg} / \mathrm{ml}$} \\
\hline 1 & $19.0 \pm 0.00$ & $20.1 \pm 0.12$ & $23.0 \pm 0.17$ & $18.5 \pm 0.07$ & $13.2 \pm 0.25$ & $15.7 \pm 1.4$ \\
\hline 2 & $12.1 \pm 0.17$ & $14.1 \pm .05$ & $20.1 \pm 0.04$ & $18.1 \pm 0.25$ & $11.0 \pm 0.77$ & $14.7 \pm 0.25$ \\
\hline 3 & $24.6 \pm 0.05$ & $20.5 \pm 0.05$ & $19.1 \pm 0.1$ & $21.7 \pm 0.44$ & NA & NA \\
\hline 4 & $11.5 \pm 1.19$ & $10.1 \pm 0.17$ & $10.0 \pm 0.11$ & $12.4 \pm 0.77$ & $11.1 \pm 0.32$ & $10.1 \pm 0.97$ \\
\hline 5 & $18.0 \pm 0.07$ & $19.0 \pm 0.24$ & $12.2 \pm 0.17$ & $12.7 \pm 0.25$ & $13.3 \pm 0.74$ & $11.6 \pm 0.54$ \\
\hline 6 & $9.09 \pm 0.17$ & $10.1 \pm 0.1$ & $10.2 \pm 0.25$ & 0.0 & $14.1 \pm 0.14$ & $16.0 \pm 0.21$ \\
\hline 7 & $9.9 \pm 0.11$ & $9.1 \pm 0.51$ & $13.4 \pm 0.14$ & NA & $13.5 \pm 1.4$ & $12.1 \pm 0.13$ \\
\hline 8 & $16.4 \pm 0.19$ & $14.4 \pm 0.12$ & $17.4 \pm 0.18$ & $20.8 \pm 0.77$ & NA & NA \\
\hline 9 & $9.8 \pm 0.15$ & $10.2 \pm 0.00$ & $11.1 \pm 0.90$ & $9.2 \pm 0.84$ & $10.4 \pm 1.5$ & NA \\
\hline 10 & $10.0 \pm 0.05$ & $9.7 \pm 0.10$ & $12.7 \pm 0.25$ & NA & $11.7 \pm 0.97$ & $10.7 \pm 1.5$ \\
\hline 11 & $8.9 \pm 0.01$ & NA & $10.1 \pm 0.05$ & $12.0 \pm 0.57$ & $16.6 \pm 1.5$ & $12.5 \pm 0.01$ \\
\hline 12 & $15.0 \pm 0.06$ & $11.1 \pm 0.11$ & NA & $13.7 \pm 0.55$ & $11.0 \pm 0.52$ & NA \\
\hline 13 & $9.5 \pm 0.01$ & $11.5 \pm 0.04$ & $9.5 \pm 0.14$ & $12.7 \pm 0.91$ & $8.5 \pm 0.97$ & NA \\
\hline 15 & $15.1 \pm .03$ & $12.2 \pm 0.51$ & $11.6 \pm 0.26$ & $13.1 \pm 0.25$ & $11.2 \pm 0.97$ & $13.4 \pm 1.2$ \\
\hline Ampicillin & $26.1 \pm 0.01$ & $21.4 \pm 0.91$ & $25.9 \pm 0.70$ & $26.0 \pm 0.81$ & NA & NA \\
\hline $\begin{array}{l}\text { Amphotericin } \\
\text { B }\end{array}$ & NA & NA & NA & NA & $17.4 \pm 0.58$ & $21.7 \pm 1.2$ \\
\hline
\end{tabular}

a: each value is the mean of three values, NA: no activity, RCMB: The Regional Center for Mycology and Biotechnology, Al-Azhar University, Cairo, Egypt

\section{Structure-activity relationship (SAR)}

Based on previously obtained antimicrobial activity of chromene derivatives, we performed the SAR studies on their activity against B. subtilis, S. aureus, E. coli and P. aeruginosa, A. flavin and C. albicans. By inspection of the experimental results of the antimicrobial activity of the synthesized chromene derivatives, the following structural activity relationship assumptions are suggested.

1-The pyrimidine moiety with incorporated with a sulfur atom in compounds 3,5 and 8 is necessary to have higher antibacterial activities towards B. subtilis, S. aureus, E. coli and P. aeruginesa.

2-It is interesting to point out that imidazole moiety in compounds 6 , 11 and 15 is necessary to have higher antifungal activity against $A$. flavin and C. albicans. The studies also emphasize the important role of cyano group in compound 1 in antimicrobial activity, which led to design new potentially active chromene, antimicrobial agents. In general, the above-mentioned results suggest that the new chromene derivatives may provide valuable leads for the synthesis and development of novel antifungal agents

\section{CONCLUSION}

In conclusion, various chromene derivatives were synthesized in efficient and easy process. Our study focused on the synthesis of new heterocyclic compounds as more powerful antimicrobial activities. In this research, we have shown the preparation procedure of new synthesized heterocyclic compounds containing a benzochromene moiety and evaluated the antimicrobial activities of all the innovative synthesized derivatives. Antimicrobial results revealed that compounds 4, 6 and 9-13, 15 showed a moderate to weak antibacterial activity against $B$. subtilis, $S$. aureus, $E$. coli and $P$. aeruginesa. In contrast, the compounds $1,6,11,15$ have higher activity towards $A$. flavin and $C$. albicans while the other compounds exhibit a moderate to weak activity. The chemical configurations of the newly synthesized compounds were clarified by spectroscopic techniques such as IR, NMR, and mass spectral data and elemental analysis. 


\section{ACKNOWLEDGMENT}

Faculty of Science, Cairo University, microanalytically center for carrying out the biological screening.

\section{AUTHORS CONTRIBUTIONS}

EMAN S. ZARIE conceived the idea, supervised the experiments, analyzed the results and drafted the manuscript. MAHMOUD $\mathrm{N}$. ABDELAZIZ performed the experiments. ALAADIN E. SARHAN contributed to the idea development.

\section{CONFLICTS OF INTERESTS}

Authors declare no conflicts of interest

\section{REFERENCES}

1. Austin DJ, Kakehashi M, Anderson RM. The transmission dynamics of antibiotic-resistant bacteria: the relationship between resistance in commensal organisms and antibiotic consumption. Proc R Soc London Ser B 1997;1388:1629-38.

2. Mwambete KD, Stephen WS. Antimicrobial resistance profiles of bacteria isolated from chicken droppings in Dar es Salaam. Int J Pharm Pharm Sci 2015;7:268-71.

3. Qingjing Y, Yanbo W, Shihui L, Jun (Joelle) W. Kinetic resolution and dynamic kinetic resolution of chromene by rhodiumcatalyzed asymmetric hydroarylation. Angew ChemInt Ed 2019;58:5343-7.

4. Hassan Y, Sarg MT, El Deeb MA, Bayoumi AH, El Rabeb SI. Facile synthesis and anticancer activity study of a novel series of substituted and fused coumarin derivatives. J Heterocycl Chem 2018;55:1426-43.

5. Safari J, Heydarian M, Zarnegar Z. Synthesis of 2-amino-7hydroxy-4H-chromene derivatives under ultrasound irradiation: a rapid procedure without catalyst. Arabian J Chem 2017;10:S2994-S3000.

6. Abd El-Wahab AHF. Synthesis, reactions and evaluation of the antimicrobial activity of some 4-(p-halophenyl)- $4 \mathrm{H}$ naphthopyran, pyranopyrimidine and pyranotriazolopyrimidine derivative. Pharmaceuticals (Basel) 2012;5:745-57.

7. Yanyang H, Rong H, Rongsheng T, Fengqiong L, Jianyou S, Mei Z. $\mathrm{K}_{2} \mathrm{CO}_{3}$ mediated synthesis of functionalized 4-substituted-2amino-3-cyano-4H-chromenes via michael-cyclization reactions. Molecules 2014;19:19253-68.

8. Hai Feng G, Wei Wei C, Zheng F, Xin LST, Kai G. Efficient synthesis of chromenopyridine and chromene via MCRS. Chinese Chem Lett 2014;25:1357-62.

9. Jian X, Yuye C, Shuaizhu LW, Lubin XU, Hongtao W. Diversified construction of chromeno[3,4-c]pyridin-5-one and benzo[c]chromen-6-one derivatives by domino reaction of 4alkynyl-2-oxo-2H-chromene-3-carbaldehyde. Adv Synth Catal 2014; 356:1835-45.

10. Isaac GS, Eugenia ML, Raguel PH. Enantioselective organocatalyzed synthesis of 2-amino-3-cyano-4H-chromene derivatives. Symmetry 2015;7:1519-35.

11. Srinivas T, Athira CK, Rajeev S. Facile synthesis of $4 \mathrm{H}$-chromene derivatives via base mediated annulation of ortho-hydroxy chalcones and 2-bromoallyl sulfones. Beilstein J Org Chem 2016;12:16-21.

12. Lamia D, Mariem K, Noureddine A, Houcine A, Souhir A. Synthesis and antibacterial activity of 2-aminochromenes a rising cyanoiminocoumarins and $\beta$-naphthol. Org Coumun 2017;10:32-9.

13. Deepali J, Anu M, Pratbha RMS, Bhartendu PT, Senehlata Y, Jaya $\mathrm{S}$, et al. A visible light-initiated, on pot, multi-component synthesis of 2-amino-4-(5-hydroxy-3-methyl-1H-pyrazol-4-yl)4-chromene-3-carbonitrile derivatives under solvent and catalyst-free conditions. Res ChemIntermed 2018;44:231-46.

14. $\mathrm{Hu} \mathrm{K}$, Lu A, Wang $\mathrm{Y}$, Zhou $\mathrm{Z}$, Tang C. Chiral bifunctionalsquaramide catalyzed asymmetric tandem michaelcyclization reaction; efficient synthesis of optically active 2 amino-4H-chromen-3-carbonitrile derivatives. Tetrahedron: Asymmetry 2013;24:953-7.

15. Vijay PP, Sunil UT, Balaji DR, Rajendra PP. Efficient and green synthesis of 2-amino-4H-chromenes. J Chem Pharm Res 2015; 7:1057-61.
16. Kamdar NR, Haveliwala DD, Mistry PT, Patel SK. Desgin, synthesis and in vitro anti-mycobacterial activity of chromene fused cytosine and uracil analogues. Anti-infect Agents Med Chem 2013;1:41-7.

17. Chetanb SN, Shah MPP, Ranjang P. Microwave assisted synthesis of novel $4 \mathrm{H}$-chromene derivatives bearing phenoxypyrazole and their antimicrobial activity assessment. J Serb Chem Soc 2012;77:1165-74.

18. El-Saghier AMM, Naili MB, Rammash Kh, Nabil AS, Khaled MK. Synthesis and antibacterial activities of some new fused chromenes. ARKIVOC 2007;16:83-91.

19. Nacy T, Subin MZ. Pharmacological activities of chromene derivatives and overview. Asian J Pharm Clin Res 2013;6:211-5.

20. Amol GJ, Suvidha SS, Dineshs P, Nagaiyan S. Phenylpyran-fused coumarin novel derivatives: a combined photophysical and theoretical study on the structural modification for PETinhibited ICT emission. Struct Chem 2018;19:217-30.

21. Masesane IB, Desta ZY. Reaction of salicylaldehyde and enolates or their equivalents: versatile synthetic routes to chromane derivatives. Beilstein J Org Chem 2012;8:2166-75.

22. Bogza YP, Katsiel AL, Sharpova AN, Tolstikova TG, Fisyuk AS. Synthesis and biological activity of $4 \mathrm{H}$-thieno[3,2-c] chromene derivative. Chem Heter Comp 2015;50:1712-8.

23. Okasha RM, Alblewi FF, Afifi TH, Naqvi A, Fouda AM, Al-Dies $\mathrm{AM}$, et al. Design of new benzo[h]chromene derivatives: antitumor activities and structure-activity relationships of the 2,3-positions fused rings at the 2,3-positions. Molecules 2017;22:479-96.

24. El Azab IH, Mohamed MY, Mahmoud AA. Microwave-assisted synthesis of novel $2 \mathrm{H}$-chromene derivatives bearing phenyl thiazolidinones and their biological activities assessment. Molecules 2014;19:19648-64.

25. Balalaie S, Bararjanian M, Sheikh Ahmadi M, Hekmat S, Salehi P. Diammonium hydrogen phosphate: an efficient and versatile catalyst for the one-pot synthesis of tetrahydrobenzo[b]pyran derivatives in aqueous media. Synth Commun 2007;37:1097-108.

26. Abbas S, Radineh M, Omidreza F, Savis M, Ahmad R, Ramin M. Synthesis and cytotoxic activity of novel benzopyrano [3,2c]chromene-6,8-dione derivatives. Med Chem Res 2011;20:466-74.

27. Reddy RK, Rao SP, Dev JG, Poornachandra Y, Ganesh Kumar C, Rao SP, et al. Synthesis of novel 1,2,3-triazole/isoxazole functionalized $2 \mathrm{H}$-chromene derivatives and their cytotoxic activity. Bioorg Med Chem Lett 2014;24:1661-3.

28. Chen JJ, Wang TY, Hwang TL. Neolignans. acoumarinolignan, lignan derivatives, and a chromene: anti-inflammatory constituents from Zanthoxylum avicennae. J Nat Prod 2008;71:212-7.

29. Fang JH, Yuan QS, Jing S, Jian BC, Hai JZ, Cai XY. Colorimetric detection of thiols using a chromenemolecule. Org Lett 2009;11:4918-21.

30. Mladenovic M, Vukovic N, Sukdolak S, Solujic S. Design of novel 4-hydroxy-chromene-2-one derivatives as antimicrobial agents. Molecules 2010;15:4294-308.

31. Serbetci T, Birteksoz S, Prado S, Michel S, Tillequin F. Synthesis and antimicrobial activities of some sulfur-containing chromene derivatives. Nat Prod Commun 2012;7:891-4.

32. Vukovic N, Sukdolak S, Solujic S, Niciforovic N. Substituted imino and amino derivatives of 4-hydroxycoumarins as a novel antioxidant, antibacterial and antifungal agents: synthesis and in vitro assessments. Food Chem 2010;120:1011-8.

33. Fadda AA, Berghot MA, Amer FA, Badawy DS, Bayoumy NM. Synthesis and antioxidant and antitumor activity of novel pyridine, chromene, thiophene and thiazole derivatives. Arch Pharm (Weinheim) 2012;345:378-85.

34. Mori J, Iwashima M, Takeuchi M, Saito H. A synthetic study on antiviral and antioxidativechromene derivative. Chem Pharm Bull 2006;54:391-6.

35. Naga Sudha B, Sridhara C, Girija Sastry C, Reddy YSR, Sreevidya 0 , Lavanya S, et al. Synthesis, characterization and anthelmintic activity of 3-(4-acetyl-5-phenyl-4,5-dihydro-1,3,4-oxadiazol-2yl)-2H-chromen-2-one derivatives. Indian J Chem 2013;52B:422-7. 
36. Bhavsar D, Trivedi J, Parekh S, Savant M, Thakrar S, Bavishi A et al. Synthesis and in vitro anti-HIV activity of $\mathrm{N}-1,3-$ benzo[d]thiazol-2-yl-2-(2-oxo-2H-chromen-4-yl)acetamide derivatives using MTT method. Bioorg Med Chem Lett 2011;21:3443-6.

37. Cheng JF, Ishikawa A, Ono Y, Arrhenius T, Nadzan A. Novel chromene derivatives as TNF-alpha inhibitors. Bioorg Med Chem Lett 2003;3(13, Suppl 21):3647-50.

38. Sun W, Cama LD, Birzin ET, Warrier S, Locco L, Mosley R, et al. 6H-Benzo[c]chromen-6-one derivatives as selective ERbeta agonists. Bioorg Med Chem Lett 2006;16:1468-72.

39. Hussain MK, Ansari MI, Yadav N, Gupta PK, Gupta AK, Saxena R, et al. Design and synthesis of $\operatorname{ER} \alpha / \operatorname{ER} \beta$ selective coumarin and chromene derivatives as potential anti-breast cancer and antiosteoporotic agents. RSC Adv 2014;4:8828-45.

40. Mungra DC, Patel MP, Rajani DP, Patel RG. Synthesis and identification of $\beta$-aryloxyquinolines and their pyrano[3,2c]chromene derivatives as a new class of antimicrobial and antituberculosis agents. Eur J Med Chem 2011;46:4192-200.

41. Vijaya KP, Rajeswar RV. Synthesis and antitubercular, antiviral and anticancer activity of 3-(3-mercaptoalkyl-7H-[1,2,4] triazolo[3,4-b][1,3,4]-thiadiazin-6-yl) chromen-2-one and its derivatives. Indian J Chem 2008;47B:106-11.

42. Bin L, Long Guan X, Xiao Hua X, Yong Hong L. Synthesis, crystal structure and herbicidal activity of 3-benzoyl-4hydroxycoumarin derivatives. Chin J Org Chem 2011;31:2067-73.

43. Hegab MI, Abdulla MM. 4-Chloro-2, 2-disubstituted chromen-3carboxaldehyde: synthesis of some fused polycyclic heterocycles as an anti-inflammatory, analgesic, anticonvulsant, and antiparkinsonian agents. Arch Pharm (Weinheim) 2006;339:41-7.

44. Ronad PM, Maddi VS, Koti BC, Kurhe YV, Swamy A, Swamy Ahmt, et al. Evaluation of the anticonvulsant activity of novel series of benzopyran-2-one derivatives by PTZ induced seizure model in mice. Indian J Novel Drug Delivery 2010;2:158-61.

45. Sivakumar KK, Rajasekaran A. Synthesis, in vivo analgesic and in vitro anti-microbial activity of 3-amino-4-[2-(substituted phenyl)hydrazin-1-ylidene]-1-[(2-oxo-2h-chromen-3-

yl)carbonyl]-4, 5-dihydro-1hpyrazol-5-one and its schiff bases. Int J Res Pharm Chem 2014;4:517-27.

46. Hegab MI, Abdulla MM. 4-Chloro-2,2-disubstituted chromen-3carboxaldehyde: synthesis of some fused polycyclic heterocycles as anti-inflammatory, analgesic, anticonvulsant, and antiparkinsonian agents. Arch Pharm (Weinheim) 2006;339:41-7.

47. Ronad PM, Maddi VS, Koti BC, Kurhe YV, Swamy A, Swamy Ahmt, et al. Evaluation of the anticonvulsant activity of novel series of benzopyran-2-one derivatives by PTZ induced seizure model in mice. Indian J Novel Drug Delivery 2010;2:158-61.

48. Patil RB, Sawant SD. Synthesis, docking studies and evaluation of antimicrobial and in vitro antiproliferative activity of $5 \mathrm{H}$ chromene 4.3-d pyrimidine-2-amine derivatives. Int J Pharm Pharm Sci 2015; 7:210-8

49. Sawsan AF, Sadia AH, Samir YA, Amel MF, Yousry AA. Synthesis of hromen-2-one, yrano[3,4-c]chromene and yridino[3,4c]chromeneerivatives as otentntimicrobial gents. Croat Chem Acta 2018;91:99-107.

50. Al-Majedy YK, Kadhum AH, Al-Amiery AA, Mohamad A. Coumarins: the antimicrobial agents. Sys Rev Pharm 2017;8:62-70.

51. Scott AC. Laboratory control of antimicrobial therapy. In: Collee JG. Eds. Practical Medical Microbiology. 13 ${ }^{\text {th }}$ edn. Edinburgh, Churchill Livingstone; 1989. p. 161-81.

52. Ramadan A, Mekheimer KUS. Microwave-assisted reactions: three-component process for the synthesis of 2-amino-2chromenes under microwave heating. Chinese Chem Lett 2009;2:271-4. 